Relations industrielles

Industrial Relations

\title{
Chefs ouvriers en sessions d'études
}

\section{Marcel de la Sablonière}

Volume 4, numéro 3, novembre 1948

URI : https://id.erudit.org/iderudit/1023443ar

DOI : https://doi.org/10.7202/1023443ar

Aller au sommaire du numéro

Éditeur(s)

Département des relations industrielles de l'Université Laval

ISSN

0034-379X (imprimé)

1703-8138 (numérique)

Découvrir la revue

Citer cet article

de la Sablonière, M. (1948). Chefs ouvriers en sessions d'études. Relations

industrielles / Industrial Relations, 4(3), 23-25. https://doi.org/10.7202/1023443ar

Tous droits réservés @ C Département des relations industrielles de l’Université Laval, 1948
Ce document est protégé par la loi sur le droit d'auteur. L'utilisation des services d'Érudit (y compris la reproduction) est assujettie à sa politique d'utilisation que vous pouvez consulter en ligne.

https://apropos.erudit.org/fr/usagers/politique-dutilisation/ 
lisme affaibli, l'opération se solderait par un recul douloureux.

Tel est le problème. A notre sens, le poser, c'est le résoudre. En effet, tous ces dangers n'existent que dans la mesure où leur crainte même inspirera aux officiers une attitude de passivité.

Mais cela n'est ni nécessaire, ni fatal. $\mathrm{Si}$, au contraire, le monde du travail, tout en respectant la liberté de l'initiative patronale, fait suffisamment connaître qu'il l'accueillerait avec faveur, le geste du patron se présentera comme une réponse, qui loin de nuire aux syndicats et aux unions les rejoindront dans une aspiration commune à la paix sociale. Lorsqu'il y a revendication de salaire, et transaction patronale, nous sommes en régime libéral. Mais si le monde du travail voit une aspiration qui lui est chère spontanément satisfaite par le patronat, en vue de réaliser une communauté au sein de l'entreprise, le syndicalisme est alors, en pleın, dans son rôle de progrès social, - nous sommes en régime chrétien. C'est ce qu'a admirablement exposé le président de la C.T.C.C., M. Gérard Picard, dans son discours d'ouverture du Congrès de 1948.

En second lieu, la prise en charge par les syndicats de l'étude de ces réformes aboutira normalement à faire d'eux au sein de l'entreprise, l'organisme d'exécution en collaboration avec la direction. Si la communauté de profit appelle, d̀ plus ou moins longue échéance, les comités d'entreprise, il est évident que seule la représentation syndicale peut donner à ces derniers une base suffisamment compétente et une suffisante maturité. En un tel cas, c'est la vigilance syndicale qui permettra d'éviter des confusions entre les ajustements du salaire de base, et la participation aux profits.

Ainsi, les dangers des réformes de l'entreprise ne sont sérieux que pour un syndicalisme passif ou hostile. Pour un syndicalisme progressif, elle peuvent être l'occasion providentielle de faire, entreprise par entreprise, le saut définitif qui permettra de passer de la renvendication à la collaboration, de l'opposition systématique à la composition systématique, de l'entreprise libérale à la libre entreprise. Et le caractère spontané de l'initiative patronale, sa valeur de soumission aux conseils pontificaux, sa portée en matière de collaboration patronale-ouvrière garantissent suffisamment aux patrons qui ont résolu dans leur coeur de "reconstruire l'édifice social sur l'inébranlable centre de gravité de la loi divine ${ }^{3}$ que de telles réformes sont les mesures les plus aptes à faire disparaître une méfiance ouvrière qui est finalement la mesure de leur propre méfiance. Seule, la Charité ne craint point.

(3) Pie XII, Message de Noël 1942.

\section{CHEFS OUVRIERS EN SESSIONS D'ÉTUDES}

Marcel de la Sablonnikere

Chaque fin de semaine du mois d'août dernier, on a vu arriver à l'île Saint-Ignace, des principaux centres industriels de la province, une quarantaine de chefs ouvriers, délégués officiels de leurs syndicats respectifs.

L'atmosphere. - Du vendredi soir au dimanche soir, aux conférences comme dans les discussions de groupes, dans les simples jasettes comme dans les forums du soir, c'était toujours la même atmosphère sympathique, toute de simplicité, de cette cordialité franche qui, toile de fond dans les rapports des ouvriers entre eux, en fait le charme et le bonheur.

Ce que l'on sentait chez tous, c'était ce grand désir de mieux connaître les réponses aux problèmes économiques et sociaux que posent dans leur vie de travailleurs organisés la condition actuelle de l'entreprise, ies pulsations alarmantes de la hausse du coût de la vie, avec ses pénibles répercussions sur la subsistance même de la famille ouvrière.

Le sujet de cette année. - Les chefs ouvriers avaient choisi comme sujet d'étude: le travailleur canadien devant l'inflation. « Ce n'est pas là, disait le P. Jacques Cousineau, S.J., directeur-fondateur de ces Sessions syndicales d'été, une question proprement syndicale ou, si l'on veut, elle ne touche pas à l'ouvrier en tant que producteur dans ses rapports avec des patrons. C'est le consommateur qui est ici en cause, c'est-à-dire l'ouvrier qui doit s'abriter, se nourrir, équilibrer un budget. »

Discussions en groupes. - Le cours terminé, - il y en a deux par jour - l'on se disperse par groupes de cinq ou six, à l'ombre bienfaisante des pins ou des mélèzes, sur la falaise, face au fleuve. 
Et c'est alors que commence le travail bien typique de ces sessions.

Notes de cours en main, l'esprit orienté vers tel ou tel aspect du problème qui l'a intéressé particulièrement, lui, ouvrier de la Chemicals (Shawinigan), ou lui, de la Wabasso (Trois-Rivières), ou lui, de l'Empire Shirt (Louiseville) . . ., ils ont cette aisance enviable de plonger immédiatement dans le concret de leur situation respective et de soulever ainsi, simplement, une déficience à corriger chez eux, un regret, une ambition, ou encore une réussite récente dans tel secteur de leur milieu de travail.

Après une heure de cet échange enrichissant, le secrétaire du groupe dresse sommairement le bilan des observations, objections, propositions suscitées et en informera le conférencier au cercle général qui suivra.

Budget familial. - M. Gérard Picard, président général de la C.T.C.C., vient d'établir devant eux le budget mensuel, hebdomadaire et annuel d'une famille-type de cinq personnes (père, mère et trois enfants), au début de l'année 1948.

Logement, chauffage et éclairage, nourriture, habillement: total par semaine, \$40; par mois, $\$ 176$; par année, $\$ 2,080$. Et cela, en se basant sur le témoignage du statisticien du Dominion, qui, comparaissant devant le Comité d'Enquête sur les Prix, déclarait lui-même que ces chiffres étaient trop bas.

Avant la guerre, à la date du mois d'août 1939, une famille moyenne pouvait se procurer les quelque 150 articles essentiels à la vie avec un revenu de $\$ 1,450$ par année.

Au début de janvier 1948, les compilations officielles fixaient à 151.3 l'indice du coût de la vie, en prenant comme base de 100 le mois d'août 1939.

Il fallait alors voir nos ouvriers comparer à cette hausse du coût de la vie l'augmentation plus ou moins parallèle qui leur est octroyée dans l'échelle de leurs salaires. Et chacun d'y aller de ses réflexions, de ses chiffres, de son budget familial. Et tous, avec un revenu parfois inférieur à celui cité plus haut, n'avaient pas toujours à leur charge une famille qui pouvait entrer dans la catégorie de la famille-type de cinq, telle celle de ce menuisier de Nicolet qui avait laissé à la maison ses 16 enfants, dont . . . 14 filles.

L'habitation à bon marché, la formule coopérative. - Au cercle général sur l'habitation à bon marché, le conférencier, M. Jean Deschamps, professeur aux Hautes Etudes commerciales eut fort à faire pour répondre au flot de questions que son exposé avait provoquées. C'était le rêve de chaque travailleur, ambition légitime de posséder une maison bien à soi, que l'on sentait passer à travers les interpellations vivantes, les demandes de précisions sur l'aspect technique de la formule coopérative, sur tout le parti que l'on peut tirer de la récente Loi provinciale du logement.

Un délégué des Trois-Rivières, racontant les merveilleuses réussites de la Coopérative d'habitation du chanoine Chamberland, fait briller bien des yeux. Un autre, de Drummondville, met la sienne en avant, avec une légitime fierté. Il en fait partie, et attend d'entrer dans sa maison sous peu ... Une délégation invite le conférencier à venir exposer cette idée merveilleuse aux membres de leur syndicat et les aider à réaliser ce rêve . . .

L'organisation de la consommation. - Même attention soutenue, même engagement à fond, dans les autres sujets. La fin de la vie économique est la consommation. Le consommateur, qui devrait dominer la vie économique, en est en réalité le jouet.

Devant un producteur et un distributeur fortement organisés, y a-t-il moyen pour le consommateur d'influencer les prix? Action syndicale? Intervention de l'Etat? Ligues de consommateurs? Arbitrages des prix? Coopératives?

La discussion s'engage, que le conférencier, M. Jean Marchand, secrétaire de la C.T.C.C., guide d'une main experte. On en jasera encore au cours du dîner qui suit.

La réforme de l'entreprise . . . - Ou bien c'est au sein de l'entreprise elle-même que les esprits se dirigent, intéressés à l'extrême par une participation possible des travailleurs à la gestion, aux bénéfices, à la propriété de lindustrie. Le $P$. Jacques Cousineau, conseiller moral du Conseil central de Montréal, en montre la convenance et la justice, développe différents modes d'application.

Le forum du soir. - Le soir, dans la douce quiétude de la nuit envahissante qu'accusent de plus en plus les feux de cigarettes, on demande à l'un ou à l'autre des délégués, président de syndicat, agent d'affaires, de faire part à tous les confrères, réunis là en rond, des expériences de l'année, succès ou insuccès, plans d'avenir.

Il y a toujours matière à causer, et l'on y va rondement, sans cérémonie, pour raconter parfois des histoires palpitantes d'intérêt. Et parce que venant de villes différentes, d'industries différentes, la situation n'est jamais la même, sauf peutêtre en ce qui a trait à la détermination, au courage, au dévouement inlassable de ces meneurs d'hommes, de ces chefs. 
Expériences. - Telle l'histoire de sa vie de jeune travailleur que nous raconte cet ancien jociste de vingt-cinq ans. Il y a trois ans, assez bien préparé à l'action par le cercle d'études local de la J.O.C., il décide un jour d'organiser en syndicat les 150 employés de l'usine où il travaille. Les raisons pour le faire sont excellentes, et . . . ça presse.

En trois mois, il a rallié à la bonne cause plus des deux tiers de ses compagnons de travail, obtient de Québec l'incorporation officielle de son syndicat, entame des négociations avec le patron. Vains efforts. Pressions de toutes sortes, congédiements ... .

Vient un conciliateur. Arbitrage. Grève qui dure plus de deux mois. Sympathie émouvante de la population pour les grévistes et leur jeune chef. Victoire finale du syndicat et de meilleures conditions de travail pour ces 150 travailleurs, dont un grand nombre chargés de famille, et cela grâce à l'initiative, à la constance et au formidable travail d'un jeune homme de vingt-cinq ans.

Un autre, doyen celui-là d'une industrie chimique de Shawinigan, évoque le triste sort de quelques-uns de ses camarades que leur travail, dans une atmosphère chargée d'émanations d'oxyde de mercure, rend, au bout de quelques années, inaptes à toute tâche, à la charge de leurs familles, détraqués qu'ils sont dans leur système nerveux, profondément intoxiqués. Ce sera l'effort principal de leur syndicat, cette année, que de chercher à faire améliorer la Loi des accidents de travail, au chapitre des maladies industrielles.

Des têtes fortes... des réactionnaires....P Trop de gens jugent encore l'ouvrier, ses chefs, ses revendications, d'une façon sommaire et souvent fort injuste. Dès qu'ils entendent parler d'unions ouvrières, de chefs ouvriers, de rajustement de salaires, de contrats collectifs, de grèves, ils voient «rouge » et crient à la domination croissante de la classe ouvrière.

« Franchement, les ouvriers exagèrent, disent ces bonnes gens. Ils n'en ont jamais assez. Ils finiront par tout mener. La hausse du coût de la vie? mais ce sont les ouvriers qui la provoquent continuellement avec leurs demandes incessantes d'augmentation de salaires. » Et l'on part ainsi... pour la gloire! Et l'on ne s'arrête pas à penser qu'il peut exister d'autres causes aux fluctuations énervantes des prix, par exemple: les variations du marché, ia rareté ou l'abondance de la production, les appétits insatiables des cartels ...

S'il est une impression forte que nous gardons de notre séjour à l'île Saint-Ignace, en contact étroit avec ces chefs de nos Syndicats nationaux, c'est bien que cet élément important de notre population canadienne-française est pondéré, raisonnable, éclairé.

A les entendre discuter calmement entre eux des problèmes que posait le programme en cours, il y avait de quoi être fier et . . . rassuré en même temps.

Qu'ils fussent de Montréal, de Chambly, des Trois-Rivières, de Sorel, de Nicolet, de La Tuque, de Shawinigan, de Joliette, de Drummondville, de Québec, de l'Epiphanie..., tous manifestaient, avec le profond désir de travailler de toutes leurs forces au relèvement de la classe ouvrière du pays, le souci constant d'accomplir cette tâche auguste dans le respect de l'ordre et de la justice.

Que Dieu bénisse le voeu formulé tant de fois par les délégués et si cher au coeur du P. Cousineau: voir bientôt se réaliser en permanence un Collège du Travail qui préparera au pays ses sauveurs, «pour empêcher que l'ouvrier ne soit condamné, selon le mot de Pie XII, à une servitude économique, inconciliable avec les droits de sa personne».

(Ma Paroisse)

INDEX DES PRODUITS ET MANUFACTURIERS DE LA PROVINCE DE QUEBEC (1948): La septième édition annuelle de cette publication bilingue vient de paraître. Elle est destinée à mieux faire connaître la diversité de la production industrielle québécoise et à favoriser des nouveaux contacts commerciaux sur le marché canadien et à l'extérieur de nos frontières. En plus de contenir des renseignements précis sur l'activité économique du Québec, cette revue illustre à l'aide de statistiques bien présentées le rôle prépondérant joué par le Québec dans l'industrie canadienne.

Indispensable à tous les hommes d'affaires, cet annuaire industriel est en vente chez l'éditeur "Les Editions du Commerce Inc.", 79 Boulevard Charest, Québec.

\section{Réfarmes de Structure dans l'Entreprise \\ Reproduction des articles publiés dans le Bulletin des relations industrielles par MM. P,-E. Bolté, Marcel Clément et Gérard Dion. Brochure d'environ 150 pages qui sera mise en vente prochainement par le Département des relations industrielles.}

